\title{
Avaliação da qualidade de vida em pacientes idosos antes e após a cirurgia de catarata
}

\author{
Quality of life assessment in elderly patients before and after cataract surgery
}

Valéria da Silva Faria ${ }^{1}$ (1), Leonardo Luiz Borges $^{2}$ (1), Jacqueline Andréia Bernardes Leão Cordeiro ${ }^{1}$ (1), Antonio Márcio Teodoro Cordeiro Silva² ${ }^{2}$, Rogério José de Almeida ${ }^{2}$ (])

Descritores:

Extração de catarata; Participação social; Qualidade de vida; Saúde do idoso

Keywords: Cataract extraction; Social participation; Quality of life; Health of the elderly

Submetido: $8 / 7 / 2020$

Aceito: $15 / 4 / 2021$

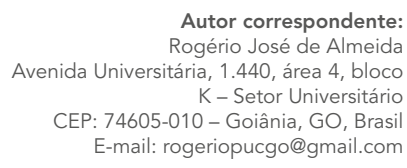

Autor correspondente: Rogério José de Almeida Avenida Universitária, 1.440, área 4, bloco K - Setor Universitário CEP: 74605-010 - Goiânia, GO, Brasil E-mail: rogeriopucgo@gmail.com

Instituição: Pontifícia Universidade Católica de Goiás Goiânia (GO), Brasil.

Fonte de auxílio à pesquisa: não financiado.

Conflito de interesses: não há conflitos de interesses

Trata-se de pesquisa desenvolvida em Dissertação de Mestrado do Programa de Pós-Graduação em Ciências Ambientais e Saúde da Pontifícia Universidade Católica de Goiás.

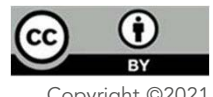

Copyright $@ 2021$

\section{RESUMO}

Objetivo: Avaliar a qualidade de vida de pacientes idosos antes e após de se submeterem à cirurgia de catarata. Métodos: Estudo longitudinal analítico com abordagem quantitativa. A coleta de dados se deu em duas etapas (antes da cirurgia de catarata e 2 meses após o procedimento), tendo sido aplicados dois questionários, sendo um sociodemográfico e o outro o World Health Organization Quality of Life OLD. Recorreu-se à estatística descritiva e aos testes estatísticos $t$ de Student, análise de variância, de Mann-Whitney e de Kruskal-Wallis. Resultados: Participaram da pesquisa 34 idosos, sendo 15 (44,1\%) até 69 anos de idade e 19 (55,9\%) com mais de 69 anos. Identificou-se decréscimo nos escores do Funcionamento do Sensório nos idosos que não tinham atividade de lazer $(p=0,0006)$, não praticavam atividade física $(p=0,0023)$ e não utilizavam a internet $(p=0,0010)$. No domínio Atividades Passadas, Presentes e Futuras, identificou-se aumento do escore nos idosos que não tinham atividade de lazer $(p=0,0258)$, não praticavam atividade física $(p=0,0303)$, afirmaram ter outros problemas de saúde $(p=0,0372)$ e referiram ter boa saúde $(p=0,0298)$. Já no domínio Participação Social, houve aumento do escore nos idosos que não tinham atividade de lazer $(p=0,0068)$, não praticavam atividade física ( $p=0,0033)$ e afirmaram ter saúde regular $(p=0,0102)$. Conclusão: Evidenciaram-se decréscimo no Funcionamento do Sensório e aumento nas Atividades Passadas, Presentes e Futuras e na Participação Social.

\section{ABSTRACT}

Objective: To evaluate quality of life of elderly patients before and after cataract surgery. Methods: A longitudinal analytical study with quantitative approach. Data collection was performed in two stages (before cataract surgery and 2 months after the procedure), when two questionnaires were used - a sociodemographic and the World Health Organization Quality of Life (WHOQLD). Descriptive statistics and Student's t test, analysis of variance, Mann-Whitney and Kruskal-Wallis tests were employed. Results: A total of 34 elderly individuals participated in the research, $15(44.1 \%)$ aged up to 69 years and $19(55.9 \%)$ over 69 years. A decrease in the Sensory Functioning scores was identified in those who had no leisure activity $(p=0.0006)$, did not exercise $(p=0.0023)$ and did not use the internet $(p=0.0010)$. In the domain Past, Present and Future Activities, an increase in the score was observed in the elderly who had no leisure activity $(p=0.0258)$, did not exercise $(p=0.0303)$, reported having other health problems $(p=0.0372)$, and in those who reported having good health status $(p=0.0298)$. In the Social Participation domain, there was an increase in the score of the elderly who had no leisure activity $(p=0.0068)$, did not exercise $(p=0.0033)$, and of those who reported having regular health status $(p=0.0102)$. Conclusion: There was a decrease in Sensory Functioning scores and an increase in the domains Past, Present and Future Activities, as well as Social Participation. 


\section{INTRODUÇÃO}

Durante o transcurso dos anos, o organismo humano se depara com diversas mudanças fisiopatológicas, que são inerentes ao processo de envelhecimento. Há, normalmente, o aparecimento de doenças relacionadas à idade. Assim, o processo de envelhecimento representa a consequência ou os efeitos da passagem do tempo no organismo e no psiquismo. ${ }^{(1)}$

O envelhecimento populacional é um fato crescente. Estima-se que, no mundo, uma a cada nove pessoas tem mais de 60 anos de idade. Estimativas apontam que, no ano de 2050, existirão mais pessoas idosas do que menores de 15 anos de idade, o que será inédito, somando-se cerca de dois bilhões de pessoas, que corresponderão a $22 \%$ da população mundial. ${ }^{(2)}$

No Brasil, o fenômeno do envelhecimento é caracterizado pela concentração de incapacidades progressivas na vida diária e nas atividades funcionais, agregada a condições socioeconômicas críticas. ${ }^{(3)}$ Nesse contexto, o ritmo veloz de envelhecimento no país tende a criar desafios novos, já que o referido processo dá-se em um panorama de profundas mudanças familiares e sociais. ${ }^{(4)} \mathrm{O}$ desgaste do organismo, individualmente, já reduz a capacidade funcional da pessoa, de maneira que as enfermidades crônicas tendem a estimular esse processo, notadamente se não houver assistência integral e multidisciplinar. ${ }^{(5)}$

A velhice é, corriqueiramente, acompanhada de perdas decorrentes do curso vital, sendo que um dos primeiros sistemas a sofrer com a ação do tempo é particularmente o visual, e o agravo mais frequente na população idosa é a catarata. ${ }^{(5)}$ Estima-se que 51\% dos casos de cegueira no mundo sejam causados pela catarata, o que significa, aproximadamente, 20 milhões de indivíduos. Ao se considerar a tendência de aumento na expectativa de vida da população mundial, verifica-se a tendência de crescimento da quantidade de pessoas que têm catarata, representando, então, um relevante motivo de baixa visão em nações em desenvolvimento e desenvolvidas. ${ }^{(3)}$

A catarata eleva a probabilidade de indivíduos da população idosa sofrerem quedas, uma vez que, em paralelo, sobrevêm a redução da percepção de distâncias, da profundidade, do equilíbrio, e a diminuição da estabilidade e da adaptação ao escuro, o que gera problemas para a percepção de perigos eminentes. (3) $^{(3)}$

Quanto ao idoso acometido pela catarata, a aferição da qualidade de vida demanda adição de variadas vertentes. Tal conceito reporta-se a distintos paradigmas que têm inspirado as rotinas de saúde. Para tanto, é preciso compreender o processo saúde-doença vinculado a vertentes socioculturais, econômicas, pessoais e os modos de vida. ${ }^{(6)}$

O objetivo deste estudo foi avaliar a qualidade de vida de pacientes idosos antes e após de se submeterem à cirurgia de catarata.

\section{MÉTODOS}

Trata-se de um estudo longitudinal analítico com abordagem quantitativa. Esse é um método de pesquisa que tem por objetivo apontar um conjunto de situações que proporcionam uma visão de profundidade sobre o tema estudado e as mudanças ocorridas na lacuna do tempo. ${ }^{(7)}$

A pesquisa foi realizada por meio de questionários aplicados à amostragem por conveniência, formada por pacientes idosos de ambos os sexos de uma clínica privada especializada em oftalmologia e cirurgia de catarata da cidade de Rio Verde (GO). A coleta de dados se deu em duas etapas, entre os meses de agosto e novembro de 2018. Na primeira etapa, foram aplicados dois questionários, sendo um sociodemográfico e o outro o World Health Organization Quality of Life OLD (WHOQOL-OLD), ao paciente idoso, antes de se submeter à cirurgia de catarata. No segundo momento, foi aplicado o WHOQOL-OLD 2 meses após a cirurgia.

Foram incluídos indivíduos de ambos os sexos, com idade igual ou superior a 60 anos, portadores de catarata e aptos à cirurgia. Foram excluídos os pacientes que não realizaram a cirurgia de catarata, apresentaram défice cognitivo e não preencheram todos os itens dos questionários.

O questionário sociodemográfico visou identificar dados sociais, como sexo, idade, estado civil, escolaridade, se contava com ajuda de familiares, se possuía emprego, se a catarata atrapalhava o desenvolvimento de seu trabalho, se recebia a ajuda dos outros, há quanto tempo sentia os sintomas da doença, há quanto tempo ocorrera a última consulta oftalmológica, se existiam outros casos da doença na família, se fora feito tratamento caseiro para catarata, se sentia medo da cegueira e medo da cirurgia, dentre outros.

O WHOQOL-OLD, por sua vez, é um instrumento de avaliação da qualidade de vida desenvolvido pela Organização Mundial de Saúde (OMS), adaptado e validado no Brasil por pesquisadores da Universidade Federal de Rio Grande do Sul. Trata-se de um questionário que busca desenvolver e testar, de maneira genérica, 
a qualidade de vida em adultos idosos. Pode ser usado em uma ampla variedade de estudos, incluindo investigações transculturais, monitoramento de saúde, epidemiologia, desenvolvimento de serviços e estudos de intervenção clínica. ${ }^{(8)}$

É um instrumento composto de seis facetas; cada uma agrega quatro itens, totalizando um questionário de 24 questões. Como cada item segue a escala do tipo Likert, de 1 a 5, sendo possível atingir escore máximo de 120 pontos e mínimo de 24. Com relação à pontuação, escores altos representam menor comprometimento de fatores que compõem a qualidade de vida e escore baixos, um maior comprometimento de fatores que compõem a qualidade de vida. ${ }^{(8)}$

A faceta Funcionamento do Sensório avalia o funcionamento sensorial e o impacto das perdas das habilidades sociais na qualidade de vida. A faceta Autonomia refere-se à independência na velhice e à capacidade de tomar suas próprias decisões. A faceta Atividades Passadas, Presentes e Futuras descreve o quão satisfeito o indivíduo está em relação às conquistas da vida e aos seus anseios. A faceta Participação Social faz um delineamento das atividades do quotidiano inserido na comunidade. A faceta Morte e Morrer relaciona-se a preocupações, temores e inquietações sobre a morte e o morrer. Por fim, a faceta Intimidade avalia a capacidade do idoso em ter relações pessoais e íntimas. ${ }^{(9)}$

Com os dados coletados, foi confeccionado um banco de dados utilizando o software IBM Statistical Package for Social Sciences, versão 18. Posteriormente, foi realizada a estatística descritiva, com o cálculo de medidas de tendência central para as variáveis contínuas, como média, e cálculo das frequências absoluta e relativa percentual, para as variáveis discretas, bem como de medidas de dispersão, como desvio-padrão.

Na sequência, foi aplicado um teste de normalidade (Kolmogorov-Smirnov) para distinguir as distribuições paramétricas e não paramétricas, com o intuito de comparação dos resultados do questionário estratificado pelas variáveis sociodemográficas. Foram utilizados, para as distribuições paramétricas, o teste $t$ de Student e a análise de variância (ANOVA) e, para as distribuições não paramétricas, o teste de Mann-Whitney e o teste de KruskalWallis. Para todos os testes comparativos, assumiu-se valor de $\mathrm{p} \leq 0,05$ como significativo.

Antes de iniciar a coleta de dados, o presente trabalho foi encaminhado ao Comitê de Ética e Pesquisa (CEP) da Pontifícia Universidade Católica de Goiás (PUC Goiás), onde foi aprovado, com parecer 2.404.484.

\section{RESULTADOS}

Participaram da pesquisa 34 idosos, sendo 15 (44,1\%) até 69 anos de idade e 19 (55,9\%) com mais de 69 anos. A menor idade foi 60 e a maior, 82. Já a média de idade foi 70,6 $( \pm 5,6)$ anos. Foram pesquisados 18 (52,9\%) homens.

As características principais identificadas na amostra investigada mostraram que 79,4\% tinham religião; 70,6\% tinham renda pessoal de até um salário mínimo; 58,8\% eram casados; 91,2\% moravam com a família e 76,5\% eram aposentados (Tabela 1).

Tabela 1. Caracterização sociodemográfica dos 34 idosos que se submeteram à cirurgia de catarata

\begin{tabular}{lc}
\hline Variáveis & $\mathbf{n}(\%)$ \\
\hline Idade, anos & $15(44,1)$ \\
\hline Até 69 & $19(55,9)$ \\
\hline Mais de 69 & \\
Sexo & $16(47,1)$ \\
\hline Feminino & $18(52,9)$ \\
\hline Masculino & \\
\hline Cor/etnia & $13(38,2)$ \\
\hline Branco & $2(5,9)$ \\
\hline Negro & $19(55,9)$ \\
\hline Pardo & \\
\hline Religião & $7(20,6)$ \\
\hline Não & $27(79,4)$ \\
\hline Sim & \\
\hline Renda pessoal mensal & $24(70,6)$ \\
\hline Até 1 salário mínimo & $10(29,4)$ \\
\hline 1-3 salários mínimos & \\
\hline Estado civil & $2(5,9)$ \\
\hline Solteiro & $20(58,8)$ \\
\hline Casado & $9(26,5)$ \\
\hline Viúvo & $3(8,8)$ \\
\hline Divorciado & \\
\hline Você mora & $3(8,8)$ \\
\hline Sozinho & $31(91,2)$ \\
\hline Com a familia & \\
\hline Escolaridade & $9(26,5)$ \\
\hline Analfabeto & $10(29,4)$ \\
\hline Sabe ler e escrever & $6(17,6)$ \\
\hline Ensino fundamental & $9(26,5)$ \\
\hline Ensino médio & \\
\hline Ocupação & $26(76,5)$ \\
\hline Aposentado & $4(11,8)$ \\
\hline Empregado & $4(11,8)$ \\
\hline Desempregado & \\
\hline & \\
\hline
\end{tabular}

No que se refere às características pessoais da amostra investigada, 79,4\% não tinham atividade de lazer, 70,6\% não praticavam atividade física e 88,2\% não usavam a internet.

Quanto aos aspectos clínicos, 76,5\% possuíam outros problemas de saúde 91,2\% tinham os sintomas da catarata há mais de 1 ano. Todos os participantes referiram que a catarata atrapalhava os afazeres diários e medo de ficar cegos (Tabela 2). 
Tabela 2. Caracterização dos aspectos pessoais e clínicos dos 34 idosos que se submeteram à cirurgia de catarata

\begin{tabular}{|c|c|}
\hline Variáveis & n (\%) \\
\hline \multicolumn{2}{|l|}{ Atividade de lazer } \\
\hline Não & $27(79,4)$ \\
\hline Sim & $7(20,6)$ \\
\hline \multicolumn{2}{|l|}{ Atividade física } \\
\hline Não & $24(70,6)$ \\
\hline Sim & $10(29,4)$ \\
\hline \multicolumn{2}{|l|}{ Usa internet } \\
\hline Não & $30(88,2)$ \\
\hline Sim & $4(11,8)$ \\
\hline \multicolumn{2}{|l|}{ Possui outros problemas de saúde } \\
\hline Não & $8(23,5)$ \\
\hline Sim & $26(76,5)$ \\
\hline \multicolumn{2}{|l|}{ Vai ao médico } \\
\hline Sim, a cada 6 meses & $11(32,4)$ \\
\hline Sim, 1 vez ao ano & $10(29,4)$ \\
\hline Às vezes, quando sente mal & $13(38,2)$ \\
\hline \multicolumn{2}{|l|}{ Você considera sua saúde } \\
\hline Boa & $14(41,2)$ \\
\hline Regular & $19(55,9)$ \\
\hline Ruim & $1(2,9)$ \\
\hline \multicolumn{2}{|c|}{ Necessita de ajuda de outras pessoas } \\
\hline Não & $23(67,6)$ \\
\hline Sim & $11(32,4)$ \\
\hline \multicolumn{2}{|l|}{ Catarata atrapalha afazeres diários } \\
\hline Não & 0 \\
\hline Sim & $34(100,0)$ \\
\hline \multicolumn{2}{|l|}{ Tempo de sintomas } \\
\hline Menos de 1 ano & $3(8,8)$ \\
\hline Mais de 1 ano & $31(91,2)$ \\
\hline \multicolumn{2}{|l|}{ Casos de catarata na família } \\
\hline Não & $17(50,0)$ \\
\hline Sim & $17(50,0)$ \\
\hline \multicolumn{2}{|l|}{ Medo de ficar cego } \\
\hline Não & 0 \\
\hline Sim & $34(100,0)$ \\
\hline \multicolumn{2}{|l|}{ Medo da cirurgia } \\
\hline Não & $19(55,9)$ \\
\hline Sim & $15(44,1)$ \\
\hline
\end{tabular}

Em relação à caracterização dos escores de qualidade de vida e seus domínios mensurados pelo WHOQOL-OLD, antes e após a cirurgia de catarata, identificaram-se, com significância estatística, decréscimo da média do escore

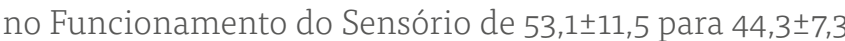
$(\mathrm{p}=0,0004)$, aumento nas Atividades Passadas, Presentes e Futuras de $67,3 \pm 10,4$ para $71,7 \pm 8,2(\mathrm{p}=0,0349)$ e aumento na Participação Social de 57,9 $\pm 11,1$ para $65,7 \pm 7,4$ $(\mathrm{p}=0,0034)$. O escore geral de qualidade de vida teve aumento de $57,4 \pm 5,9$ para $58,0 \pm 6,4$, mas sem significância estatística ( $\mathrm{p}=0,6796)$ (Tabela 3).

$\mathrm{Na}$ comparação dos escores de qualidade de vida e seus domínios com os aspectos sociodemográficos, evidenciou-se decréscimo nos escores do Funcionamento do Sensório nos idosos com idade até 69 anos de 58,3 para 44,2 ( $\mathrm{p}=0,0007)$, tanto entre as mulheres, de 53,9 para $44,2(p=0,0143)$, quanto entre os homens, de 52,4 para 44,5 $(\mathrm{p}=0,0119)$ (Tabela 4).
Tabela 3. Caracterização dos escores de qualidade de vida e seus domínios dos 34 idosos que se submeteram à cirurgia de catarata

\begin{tabular}{lccc} 
Domínios & \multicolumn{2}{c}{ Média \pm desvio padrão } & \multirow{2}{*}{ Valor de p } \\
& Antes & Depois & \\
\hline Funcionamento do Sensório & $53,1 \pm 11,5$ & $44,3 \pm 7,3$ & $0,0004^{*}$ \\
Autonomia & $54,8 \pm 10,4$ & $53,9 \pm 11,8$ & $0,7363 \dagger$ \\
Atividades Passadas, Presentes e Futuras & $67,3 \pm 8,7$ & $71,7 \pm 8,2$ & $0,0349 \dagger$ \\
Participação Social & $57,9 \pm 11,1$ & $65,7 \pm 7,4$ & $0,0034^{*}$ \\
Morte e Morrer & $40,3 \pm 27,3$ & $41,2 \pm 27,3$ & $0,8909 \dagger$ \\
Intimidade & $71,0 \pm 11,6$ & $71,3 \pm 11,2$ & $0,8955 \dagger$ \\
Qualidade de vida geral & $57,4 \pm 5,9$ & $58,0 \pm 6,4$ & $0,6796 \dagger$ \\
\hline
\end{tabular}

*Teste de Mann-Whitney; tteste $t$ de Student.

No domínio Atividades Passadas, Presentes e Futuras identificou-se aumento do escore nos idosos que tinham religião de 68,3 para 72,9 ( $\mathrm{p}=0,0263)$, nos que moravam com a família de 67,2 para $71,6(\mathrm{p}=0,0264)$, naqueles que afirmaram ser analfabetos de 66,0 para 70,2 ( $p=0,0011$ ) e nos que referiram receber até um salário mínimo mensal de 66,4 para $71,6(\mathrm{p}=0,0215)$.

Já no domínio Participação Social, houve aumento do escore nos idosos que tinham mais de 69 anos de 57,9 para $65,2(\mathrm{p}=0,0410)$, nas mulheres de 55,9 para 64,9 $(\mathrm{p}=0,0053)$, nos que tinham religião de 57,2 para 65,1 $(\mathrm{p}=0,0044)$, nos casados de 56,3 para $65,3(\mathrm{p}=0,0090)$, nos que moravam com a família de 57,9 para 65,8 $(\mathrm{p}=0,0050)$, nos analfabetos de 53,5 para 62,5 $(\mathrm{p}=0,0341)$ e nos que afirmaram saber ler e escrever de 55,7 para $66,9(\mathrm{p}=0,0284)$.

Na comparação dos escores de qualidade de vida e seus domínios com os aspectos pessoais e clínicos da amostra investigada, identificou-se decréscimo nos escores do Funcionamento do Sensório nos idosos que não tinham atividade de lazer de 53,0 para 43,1 ( $\mathrm{p}=0,0006)$, não praticavam atividade física de 53,1 para 43,5 (p=0,0023) e naqueles que não utilizavam a internet de 53,1 para 44,0 ( $\mathrm{p}=0,0010)$ (Tabela 5).

No domínio Atividades Passadas, Presentes e Futuras, identificou-se aumento do escore nos idosos que não tinham atividade de lazer de 67,4 para 72,0 ( $\mathrm{p}=0,0258)$, não praticavam atividade física de 66,2 para 70,6 ( $\mathrm{p}=0,0303)$, afirmaram ter outros problemas de saúde de 67,1 para 70,9 ( $\mathrm{p}=0,0372)$ e naqueles que referiram ter boa saúde de 69,7 para $75,5(\mathrm{p}=0,0298)$.

Já no domínio Participação Social, houve aumento do escore nos idosos que não tinham atividade de lazer de 56,7 para 65,3 ( $\mathrm{p}=0,0068$ ), não praticavam atividade física de 56,3 para $65,4(\mathrm{p}=0,0033)$, afirmaram ter saúde regular de 56,6 para 65,8 ( $p=0,0102$ ) e referiram que não tinham medo da cirurgia de 55,9 para 65,4 ( $\mathrm{p}=0,0025)$. 
Tabela 4. Comparação dos escores de qualidade de vida e seus domínios com aspectos sociodemográficos dos 34 idosos que se submeteram à cirurgia de catarata

\begin{tabular}{|c|c|c|c|c|c|c|c|c|c|c|c|c|c|c|c|c|c|c|c|c|c|}
\hline \multirow{2}{*}{ Variáveis ( $n=34$ ) } & \multicolumn{2}{|c|}{ FS } & \multirow{2}{*}{$\begin{array}{l}\text { Valor } \\
\text { de } p\end{array}$} & \multicolumn{2}{|c|}{ AUT } & \multirow{2}{*}{$\begin{array}{l}\text { Valor } \\
\text { de p }\end{array}$} & \multicolumn{2}{|c|}{ PPF } & \multirow{2}{*}{$\begin{array}{l}\text { Valor } \\
\text { de } p\end{array}$} & \multicolumn{2}{|c|}{ PSO } & \multirow{2}{*}{$\begin{array}{l}\text { Valor } \\
\text { de } p\end{array}$} & \multicolumn{2}{|c|}{ MEM } & \multirow{2}{*}{$\begin{array}{l}\text { Valor } \\
\text { de p }\end{array}$} & \multicolumn{2}{|c|}{ INT } & \multirow{2}{*}{$\begin{array}{l}\text { Valor } \\
\text { de } p\end{array}$} & \multicolumn{2}{|c|}{ QV geral } & \multirow{2}{*}{$\begin{array}{l}\text { Valor } \\
\text { de } p\end{array}$} \\
\hline & A & D & & A & D & & A & D & & A & D & & A & D & & A & D & & A & D & \\
\hline \multicolumn{22}{|l|}{ Idade, anos } \\
\hline Até 69 & 58,3 & 44,2 & 0,0007 & 55,4 & 53,8 & 0,8519 & 64,2 & 69,2 & 0,1103 & 57,9 & 66,3 & 0,0512 & 39,6 & 40,0 & 0,9174 & 68,8 & 68,8 & 0,9339 & 57,4 & 57,0 & 0,8035 \\
\hline Mais de 69 anos & 49,0 & 44,4 & 0,0961 & 54,3 & 54,0 & 0,9302 & 69,8 & 73,7 & 0,1484 & 57,9 & 65,2 & 0,0410 & 40,8 & 42,1 & 0,7481 & 72,7 & 73,4 & 0,8040 & 57,4 & 58,8 & 0,3427 \\
\hline \multicolumn{22}{|l|}{ Sexo } \\
\hline Feminino & 53,9 & 44,2 & 0,0143 & 53,2 & 50,4 & 0,7345 & 67,2 & 70,3 & 0,2000 & 55,9 & 64,9 & 0,0053 & 39,5 & 43,0 & 0,6109 & 69,2 & 70,7 & 0,7487 & 56,5 & 57,2 & 0,6109 \\
\hline Masculino & 52,4 & 44,5 & 0,0119 & 56,3 & 57,0 & 0,7758 & 67,4 & 72,9 & 0,0967 & 59,8 & 66,4 & 0,0967 & 41,0 & 39,6 & 0,9621 & 72,6 & 71,9 & 0,9370 & 58,2 & 58,7 & 0,7517 \\
\hline \multicolumn{22}{|l|}{ Cor/Etnia } \\
\hline Branco & 52,4 & 43,8 & 0,0096 & 55,8 & 58,7 & 0,4267 & 67,8 & 72,1 & 0,2382 & 51,0 & 63,5 & 0,0196 & 36,6 & 39,9 & 0,6629 & 72,1 & 71,2 & 0,6444 & 55,9 & 58,2 & 0,1998 \\
\hline Negro & 56,3 & 43,8 & 0,2961 & 50,0 & 50,0 & 1,0000 & 68,8 & 75,0 & 0,5000 & 59,4 & 62,6 & 0,6958 & 68,8 & 68,8 & 0,9984 & 75,0 & 75,0 & 1,0000 & 63,1 & 62,5 & 0,8526 \\
\hline Pardo & 53,3 & 44,8 & 0,0558 & 54,6 & 51,0 & 0,3972 & 66,8 & 71,1 & 0,1568 & 62,5 & 67,5 & 0,0471 & 39,8 & 39,2 & 0,9651 & 69,7 & 71,1 & 0,5692 & 57,8 & 57,4 & 0,8153 \\
\hline \multicolumn{22}{|l|}{ Religião } \\
\hline Não & 46,4 & 43,8 & 0,7494 & 54,5 & 53,6 & 0,8983 & 63,4 & 67,0 & 0,2502 & 60,7 & 67,9 & 0,3067 & 34,0 & 32,2 & 0,8983 & 71,4 & 73,2 & 0,7983 & 55,1 & 56,3 & 1,0000 \\
\hline Sim & 54,9 & 44,5 & 0,0002 & 54,9 & 54,0 & 0,9242 & 68,3 & 72,9 & 0,0263 & 57,2 & 65,1 & 0,0044 & 41,9 & 43,5 & 0,8019 & 70,8 & 70,8 & 0,9793 & 58,0 & 58,5 & 0,7753 \\
\hline \multicolumn{22}{|l|}{ Renda pessoal mensal } \\
\hline Até $1 \mathrm{SM}$ & 54,2 & 44,8 & 0,0024 & 51,8 & 51,6 & 0,8206 & 66,4 & 71,6 & 0,0215 & 58,4 & 64,9 & 0,0198 & 36,8 & 36,5 & 0,9671 & 70,6 & 71,1 & 0,8447 & 56,4 & 56,7 & 0,9179 \\
\hline De 1 a $3 \mathrm{SM}$ & 50,7 & 43,2 & 0,0410 & 61,9 & 59,4 & 0,7913 & 69,4 & 71,9 & 0,6501 & 56,9 & 67,5 & 0,0482 & 48,8 & 52,5 & 0,9097 & 71,9 & 71,9 & 1,0000 & 59,9 & 61,1 & 0,2899 \\
\hline \multicolumn{22}{|l|}{ Estado civil } \\
\hline Solteiro & 68,8 & 43,8 & 0,0781 & 62,5 & 62,6 & 0,9975 & 68,8 & 71,9 & 0,6958 & 62,5 & 71,9 & 0,5413 & 31,3 & 37,6 & 0,8787 & 75,0 & 75,0 & 1,0000 & 61,5 & 60,5 & 0,5700 \\
\hline Casado & 51,3 & 44,7 & 0,0167 & 54,1 & 54,7 & 0,7251 & 69,4 & 72,5 & 0,3867 & 56,3 & 65,3 & 0,0090 & 39,1 & 40,0 & 0,8817 & 70,3 & 70,0 & 0,9569 & 56,7 & 57,9 & 0,4094 \\
\hline Viúvo & 49,3 & 43,1 & 0,2332 & 54,2 & 48,6 & 0,3314 & 63,2 & 69,5 & 0,0318 & 59,1 & 63,9 & 0,3538 & 46,6 & 46,5 & 0,7573 & 72,9 & 75,0 & 0,6911 & 57,5 & 57,8 & 1,0000 \\
\hline Divorciado & 66,7 & 45,8 & 0,0360 & 56,3 & 58,4 & 0,8313 & 64,6 & 72,9 & 0,0474 & 62,5 & 68,8 & 0,4373 & 35,4 & 35,5 & 0,9986 & 66,7 & 66,7 & 1,0000 & 58,7 & 58,0 & 0,8270 \\
\hline Você mora & & & & & & & & & & & & & & & & & & & & & \\
\hline Sozinho & 54,2 & 43,8 & 0,1878 & 54,2 & 47,9 & 0,6927 & 68,8 & 72,9 & 0,3730 & 58,4 & 64,6 & 0,4687 & 58,4 & 56,3 & 0,9135 & 60,4 & 62,5 & 0,8524 & 59,0 & 58,0 & 0,9122 \\
\hline Com a família & 53,0 & 44,4 & 0,0011 & 54,9 & 54,5 & 0,9663 & 67,2 & 71,6 & 0,0264 & 57,9 & 65,8 & 0,0050 & 38,5 & 39,7 & 0,8054 & 72,0 & 72,2 & 0,8880 & 57,2 & 58,0 & 0,5264 \\
\hline Escolaridade & & & & & & & & & & & & & & & & & & & & & \\
\hline Analfabeto & 52,1 & 43,8 & 0,1451 & 49,3 & 47,2 & 0,7573 & 66,0 & 70,2 & 0,0011 & 53,5 & 62,5 & 0,0341 & 45,9 & 49,3 & 0,5660 & 67,4 & 68,1 & 0,9648 & 55,7 & 56,8 & 0,6588 \\
\hline Sabe ler e escrever & 52,5 & 43,2 & 0,0376 & 55,0 & 55,7 & 0,7055 & 66,9 & 72,5 & 0,2899 & 55,7 & 66,9 & 0,0284 & 37,5 & 36,9 & 0,7337 & 70,6 & 69,4 & 0,7337 & 56,4 & 57,4 & 0,5708 \\
\hline Ensino fundamental & 54,2 & 47,9 & 0,4233 & 60,4 & 57,3 & 0,6310 & 69,8 & 74,0 & 0,2980 & 66,7 & 69,8 & 0,3367 & 36,5 & 32,3 & 0,8728 & 81,3 & 80,2 & 0,8728 & 61,5 & 60,3 & 0,4233 \\
\hline Ensino médio & 54,2 & 43,8 & 0,0062 & 56,3 & 56,3 & 0,7911 & 67,4 & 70,8 & 0,3538 & 59,0 & 64,6 & 0,2893 & 40,3 & 43,8 & 0,7911 & 68,1 & 70,8 & 0,4529 & 57,5 & 58,3 & 0,8946 \\
\hline Ocupação & & & & & & & & & & & & & & & & & & & & & \\
\hline Aposentado & 50,3 & 44,3 & 0,0165 & 56,3 & 54,4 & 0,7212 & 68,3 & 71,6 & 0,1699 & 59,9 & 66,9 & 0,0157 & 40,2 & 42,1 & 0,7697 & 72,9 & 73,6 & 0,7143 & 57,9 & 58,8 & 0,5041 \\
\hline Empregado & 62,5 & 45,3 & 0,0416 & 51,6 & 51,6 & 1,0000 & 65,7 & 73,5 & 0,1489 & 56,3 & 64,1 & 0,2482 & 37,5 & 39,1 & 0,8852 & 61,0 & 61,0 & 1,0000 & 55,8 & 55,7 & 0,7728 \\
\hline Desempregado & 62,5 & 43,8 & 0,0304 & 48,5 & 53,2 & 0,2482 & & 70,4 & 0,1939 & 46,9 & 59,4 & 0,0433 & 43,8 & 37,6 & 0,5637 & 68,8 & 67,2 & 0,6650 & 55,5 & 55,2 & 1,0000 \\
\hline
\end{tabular}

Tabela 5. Comparação dos escores de qualidade de vida e seus domínios com os aspectos individuais e clínicos dos 34 idosos que se submeteram à cirurgia de catarata

\begin{tabular}{|c|c|c|c|c|c|c|c|c|c|c|c|c|c|c|c|c|c|c|c|c|c|}
\hline \multirow{2}{*}{ Variáveis } & \multicolumn{2}{|c|}{ FS } & \multirow{2}{*}{$\begin{array}{l}\text { Valor } \\
\text { de p }\end{array}$} & \multicolumn{2}{|c|}{ AUT } & \multirow{2}{*}{$\begin{array}{l}\text { Valor } \\
\text { de p }\end{array}$} & \multicolumn{2}{|c|}{ PPF } & \multirow{2}{*}{$\begin{array}{l}\text { Valor } \\
\text { de p }\end{array}$} & \multicolumn{2}{|c|}{ PSO } & \multirow{2}{*}{$\begin{array}{l}\text { Valor } \\
\text { de p }\end{array}$} & \multicolumn{2}{|c|}{ MEM } & \multirow{2}{*}{$\begin{array}{l}\text { Valor } \\
\text { de p }\end{array}$} & \multicolumn{2}{|c|}{ INT } & \multirow{2}{*}{$\begin{array}{l}\text { Valor } \\
\text { de } p\end{array}$} & \multicolumn{2}{|c|}{ QV geral } & \multirow{2}{*}{$\begin{array}{l}\text { Valor } \\
\text { de } p\end{array}$} \\
\hline & A & D & & A & D & & A & D & & A & D & & A & D & & A & D & & A & D & \\
\hline \multicolumn{22}{|l|}{ Atividade de lazer } \\
\hline Não & 53,0 & 43,1 & 0,0006 & 55,6 & 55,8 & 0,7687 & 67,4 & 72,0 & 0,0258 & 56,7 & 65,3 & 0,0068 & 37,1 & 38,9 & 0,7229 & 72,0 & 72,5 & 0,9862 & 57,0 & 57,9 & 0,4622 \\
\hline Sim & 53,6 & 49,1 & 0,4822 & 51,8 & 46,5 & 0,4433 & 67,0 & 70,6 & 0,4822 & 62,5 & 67,0 & 0,2502 & 52,7 & 50,0 & 0,8480 & 67,0 & 67,0 & 0,9491 & 59,1 & 58,4 & 0,7494 \\
\hline \multicolumn{22}{|l|}{ Atividade física } \\
\hline Não & 53,1 & 43,5 & 0,0023 & 54,7 & 55,0 & 0,7807 & 66,2 & 70,6 & 0,0303 & 56,3 & 65,4 & 0,0033 & 40,1 & 41,2 & 0,8366 & 72,9 & 73,2 & 0,8609 & 57,2 & 58,1 & 0,4579 \\
\hline Sim & 53,2 & 46,3 & 0,1041 & 55,0 & 51,3 & 0,5708 & 70,0 & 74,4 & 0,3643 & 61,9 & 66,3 & 0,2730 & 40,7 & 41,3 & 0,9699 & 66,3 & 66,9 & 0,8206 & 57,8 & 57,7 & 0,8501 \\
\hline \multicolumn{22}{|l|}{ Usa internet } \\
\hline Não & 53,1 & 44,0 & 0,0010 & 54,4 & 52,9 & 0,8534 & 66,1 & 70,4 & 0,0444 & 57,3 & 65,2 & 0,0056 & 38,8 & 39,4 & 0,9000 & 70,4 & 70,6 & 1,0000 & 56,7 & 57,1 & 0,6204 \\
\hline Sim & 53,2 & 46,9 & 0,2482 & 57,9 & 61,0 & 0,8852 & 76,6 & 81,3 & 0,3123 & 62,5 & 68,8 & 0,3123 & 51,6 & 54,7 & 0,6650 & 75,0 & 76,6 & 0,5637 & 62,8 & 64,9 & 0,6650 \\
\hline \multicolumn{22}{|l|}{ Problema de saúde } \\
\hline Não & 53,2 & 46,1 & 0,0313 & 56,3 & 57,8 & 0,6744 & 68,0 & 74,2 & 0,2701 & 61,0 & 67,2 & 0,0464 & 50,0 & 46,9 & 0,7929 & 71,9 & 71,9 & 1,0000 & 60,0 & 60,7 & 0,7929 \\
\hline Sim & 53,1 & 43,8 & 0,0039 & 54,3 & 52,7 & 0,8405 & 67,1 & 70,9 & 0,0372 & 57,0 & 65,2 & 0,0101 & 37,3 & 39,4 & 0,7349 & 70,7 & 71,2 & 0,8692 & 56,6 & 57,2 & 0,6805 \\
\hline \multicolumn{22}{|l|}{ Vai ao médico } \\
\hline Sim, a cada 6 meses & 55,1 & 46,6 & 0,1396 & 55,1 & 55,1 & 0,9738 & 69,9 & 72,2 & 0,5545 & 58,0 & 67,6 & 0,0330 & 38,7 & 39,2 & 0,9476 & 74,4 & 73,9 & 0,9738 & 58,5 & 59,1 & 0,8955 \\
\hline Sim, 1 vez ao ano & 48,8 & 43,2 & 0,2123 & 57,5 & 54,4 & 0,7055 & 68,8 & 73,1 & 0,3075 & 60,0 & 66,9 & 0,1509 & 46,9 & 50,0 & 0,8206 & 71,9 & 72,5 & 0,8206 & 59,0 & 60,0 & 0,5708 \\
\hline Às vezes & 54,8 & 43,3 & 0,0004 & 52,4 & 52,4 & 0,7389 & 64,0 & 70,2 & 0,0355 & 56,3 & 63,0 & 0,0429 & 36,6 & 36,1 & 0,9795 & 67,3 & 68,3 & 0,9795 & 55,2 & 55,5 & 0,8576 \\
\hline \multicolumn{22}{|l|}{ Sua saúde* } \\
\hline Boa & 52,7 & 44,2 & 0,1029 & 57,2 & 56,7 & 0,8722 & 69,7 & 75,5 & 0,0298 & 61,2 & 66,1 & 0,1029 & 37,5 & 38,4 & 0,7828 & 70,6 & 71,9 & 0,6459 & 58,1 & 58,8 & 0,7828 \\
\hline Regular & 54,6 & 44,8 & 0,0006 & 52,7 & 51,3 & 0,9070 & 65,8 & 69,4 & 0,2147 & 56,6 & 65,8 & 0,0102 & 40,2 & 40,8 & 0,9070 & 71,1 & 70,7 & 0,8267 & 56,8 & 57,1 & 0,8495 \\
\hline \multicolumn{22}{|l|}{ Necessita de ajuda } \\
\hline Não & 55,2 & 45,4 & 0,0012 & 55,7 & 54,1 & 0,7752 & 67,1 & 71,2 & 0,0350 & 59,8 & 67,2 & 0,0139 & 41,3 & 41,9 & 0,9475 & 70,4 & 71,2 & 0,7584 & 58,2 & 58,5 & 0,9300 \\
\hline Sim & 48,9 & 42,1 & 0,1077 & 52,9 & 53,4 & 0,9215 & 67,6 & 72,7 & 0,3246 & 54,0 & 62,5 & 0,0409 & 38,1 & 39,8 & 0,9738 & 72,2 & 71,6 & 0,7928 & 55,6 & 57,0 & 0,3579 \\
\hline Tempo de Sintomas & & & & & & & & & & & & & & & & & & & & & \\
\hline Menos de 1 ano & 43,8 & 41,7 & 0,7691 & 54,2 & 56,3 & 0,7691 & 68,8 & 68,8 & 1,0000 & 47,9 & 62,5 & 0,0456 & 50,0 & 56,3 & 0,8287 & 72,9 & 72,9 & 1,0000 & 56,2 & 59,7 & 0,3740 \\
\hline Mais de 1 ano & 54,1 & 44,6 & 0,0005 & 54,9 & 53,7 & 0,8825 & 67,2 & 72,0 & 0,0324 & 58,9 & 66,0 & 0,0088 & 39,3 & 39,7 & 0,8714 & 70,8 & 71,2 & 0,8936 & 57,5 & 57,8 & 0,8493 \\
\hline Casos na família & & & & & & & & & & & & & & & & & & & & & \\
\hline Não & 54,8 & 46,0 & 0,0114 & 51,1 & 49,7 & 0,8768 & 65,8 & 70,2 & 0,0353 & 55,9 & 63,3 & 0,0302 & 44,9 & 43,0 & 0,9588 & 69,9 & 69,5 & 0,9588 & 57,1 & 56,9 & 0,8768 \\
\hline Sim & 51,5 & 42,7 & 0,0131 & 58,5 & 58,1 & 0,9451 & 68,8 & 73,2 & 0,2486 & 60,0 & 68,0 & 0,0210 & 35,7 & 39,4 & 0,5934 & 72,1 & 73,2 & 0,8095 & 57,7 & 59,1 & 0,4384 \\
\hline Medo da cirurgia & & & & & & & & & & & & & & & & & & & & & \\
\hline Não & 52,7 & 44,4 & 0,0173 & 57,9 & 58,2 & 0,8609 & 67,4 & 71,4 & 0,1939 & 59,6 & 65,8 & 0,1218 & 33,6 & 34,2 & 0,8267 & 71,7 & 72,7 & 0,8040 & 57,1 & 57,8 & 0,5692 \\
\hline Sim & 53,8 & 44,2 & 0,0101 & 50,9 & 48,4 & 0,6482 & 67,1 & 72,1 & 0,0465 & 55,9 & 65,4 & 0,0025 & 48,8 & 50,0 & 0,7244 & 70,0 & 69,6 & 0,9504 & 57,7 & 58,3 & 0,7089 \\
\hline
\end{tabular}

FS: Funcionamento do Sensório; A: Antes; D: Depois; AUT: Autonomia; PPF: Atividades Passadas, Presentes e Futuras; PSO: Participação Social; MEM: Morte e Morrer; INT: Intimidade; QV: qualidade de vida. 


\section{DISCUSSÃO}

Dados do Instituto Brasileiro de Geografia e Estatística (IBGE) revelam que a população do Brasil manteve a tendência de envelhecimento, ${ }^{(10)}$ a qual ampliou a necessidade de pesquisas sobre qualidade de vida de pessoas da terceira idade, como a presente, com o propósito de fomentar ações que previnam e retardem condições de risco. ${ }^{(11)}$

Para tanto, a amostra investigada evidenciou que significativa parcela dos entrevistados tinha alguma religião, eram casados e moravam com a família. Nesse perfil, também se enquadram os aspectos relacionados à renda e à situação atual de exercício de atividade laboral. As informações aferidas revelam consonância com o perfil da população da terceira idade brasileira que porta doenças oculares, uma vez que, neste grupo social, há prevalência de aposentados com renda de até um salário mínimo. ${ }^{(3)}$

Tais aspectos revelam um perfil sociodemográfico da população entrevistada que possui baixa capacidade econômica, o que pode representar pouco acesso às informações de saúde e, por consequência, contribuir na diminuição da percepção pessoal de qualidade de vida.

A influência das características sociodemográficas na percepção de qualidade de vida dessa população é considerável, sobretudo pelo fato de que tais fatores podem influenciar de forma decisiva no estado de saúde, no acesso a programas de promoção de saúde e na rede de apoio social. ${ }^{(12)}$

A caracterização dos aspectos pessoais e clínicos da população entrevistada denotou força à referida hipótese, uma vez que a maior parte da amostra afirmou não praticar atividades de lazer, não praticar atividade física, não acessar a internet e sentir os sintomas da catarata por muitos meses, quando perguntados acerca de cada uma dessas variáveis.

A ausência de atividade física contribui para o decréscimo da qualidade de vida da população, o que se atesta quando diversos autores observam relação gradativa e direta entre maiores escores de qualidade de vida e frequência de atividade física. ${ }^{(13-15)}$

A natureza multifatorial do envelhecimento demonstra que a qualidade de vida sofre influências tanto de vertentes clínicas e comportamentais, quanto de fatores demográficos e socioculturais. ${ }^{(15)}$ Ao se considerar que toda a amostra do presente estudo referiu que a catarata atrapalhava os afazeres diários, observa-se convergência com tal perspectiva. ${ }^{(16)}$

No que tange à caracterização dos escores de qualidade de vida e seus domínios, constatou-se, na amostra, o decréscimo na média do Funcionamento do Sensório. O domínio do Funcionamento do Sensório é representado pela manutenção dos sentidos (audição, olfato, visão paladar e tato), de forma que a perda dessas capacidades pode comprometer a participação dos idosos em atividades e o potencial que eles têm de interagir com terceiros, tornando-os dependentes de cuidados e com consequente prejuízo de sua qualidade de vida. ${ }^{|17\rangle}$

Por isso, embora o idoso não tenha mais catarata, faz-se necessário o acompanhamento pós-cirúrgico por profissionais das mais diversas áreas da saúde, a fim de que outras eventuais condições prejudiciais à qualidade de vida do idoso também sejam alvo de intervenção, de modo a melhorar a condição de vida do indivíduo.

A intervenção cirúrgica de catarata senil permite melhora no equilíbrio, no desempenho funcional de membros inferiores e do medo de quedas em idosos. Trata-se de método importante e relevante para reduzir o risco de quedas e prevenir desequilíbrios, comuns no processo fisiológico do envelhecimento. ${ }^{(18)}$

É importante considerar a complexidade do funcionamento sensório, que é influenciado por todos os sentidos (audição, olfato, visão paladar e tato). Infere-se a possibilidade de que, mesmo com a intervenção cirúrgica em um dos sentidos humanos, os outros permaneçam prejudicados.

Foram encontrados maiores escores após a cirurgia de catarata no domínio Atividades Passadas, Presentes e Futuras. É imperioso registrar que o referido domínio é caracterizado pela percepção do idoso acerca daquelas atividades que já ele exerceu/realizou ou continua exercendo/realizando, bem como quanto ao que planeja fazer no futuro, ou seja, seus projetos e perspectivas.

A obtenção de maiores escores nesse domínio denota que a realização da cirurgia de catarata proporcionou maior satisfação dos idosos com as conquistas obtidas e esperança na concretização dos objetivos futuros. Tal fato se mostra de extrema importância e pode significar que o idoso não se sente mais impossibilitado de desempenhar as atividades que desempenhava antes da catarata, por exemplo, impactando, de maneira positiva, em suas perspectivas futuras. Quando os idosos não se sentem aptos a desempenharem atividades em virtude de problemas de saúde, há potencial impacto em suas expectativas.

De semelhante modo, observou-se aumento do escore no domínio Participação Social. Tal domínio se caracteriza pelas atividades corriqueiras, com relevo para as exercidas em comunidade. Na presente pesquisa, constatou-se que, após a cirurgia de catarata, os avaliados se 
mostraram mais satisfeitos para participarem das atividades da comunidade da qual faziam parte.

Uma vez que as relações sociais são fundamentais ao bem-estar do ser humano e estão, de maneira relevante, envolvidas com a manutenção da saúde, a comunidade deve se empenhar em ofertar práticas que favoreçam a participação social do idoso em ocasiões de lazer e eventos comunitários, cooperando para que eles se sintam aceitos e valorizados no grupo. ${ }^{(19)}$ Isso se aplica ao presente estudo na medida em que revela a importância dos relacionamentos na saúde do idoso que, após submeter-se à cirurgia de catarata, não está mais estigmatizado por não poder enxergar bem seu próximo ou o que acontece à sua volta.

É eficaz a intervenção cirúrgica realizada em face da catarata na melhoria da acuidade visual, ressaltando, inclusive, que idosos com medo de cair e que tinham catarata registram menos confiança no equilíbrio próprio, o que pode limitar as atividades funcionais. ${ }^{(20)}$ Nas mulheres, em especial, é maior a associação das quedas entre os pacientes com catarata. As mulheres apresentam cinco vezes mais chance de cair do que os homens, o que pode se dar talvez porque elas sofrem declínio motor, cognitivo e sensorial mais precocemente que eles. ${ }^{(21)}$

Embora não seja possível afirmar que a cirurgia mencionada resulte na redução do risco de quedas, ante a existência de outras variáveis que influenciam nesse possível evento, ${ }^{(20)}$ tem-se que a solução do problema visual, objeto desta pesquisa, aumenta a possibilidade do idoso se inserir em maior número de eventos sociais e de lazer, interagindo na comunidade em que está inserido.

É importante destacar que não houve relevância estatística para o escore geral, que apontou aumento na qualidade de vida após a cirurgia de catarata. Isso é compreensível na medida em que se observam as características pessoais e clínicas dos idosos avaliados. A percepção de qualidade de vida é produto de uma série de comportamentos adotados, não sendo influenciada apenas por um componente. ${ }^{(12)}$

Na comparação dos escores de qualidade de vida e seus domínios com os aspectos sociodemográficos, também se evidenciou decréscimo nos escores do Funcionamento do Sensório nos idosos com idade até 69 anos, tanto entre as mulheres quanto entre os homens.

Infere-se, também, que o acompanhamento pós-cirúrgico dos componentes da amostra por uma equipe integrada de profissionais da saúde pode ser necessário, a fim de que as demais vertentes sensoriais também sejam reestabelecidas ou tratadas.
No domínio Atividades Passadas, Presentes e Futuras, identificou-se aumento do escore nos idosos que tinham religião, moravam com a família, referiram receber até um salário mínimo mensal e nos analfabetos. A satisfação pessoal dos indivíduos-alvo da amostragem acerca da concretização da hipótese de melhoria da visão e a influência do meio social para tanto, possibilitando que referidos indivíduos desempenhem, com maior desenvoltura, as atividades que lhes permitam gozar a vida com mais qualidade. A percepção da qualidade de vida é o produto de uma série de componentes, e fatores sociais como renda, escolaridade e estado conjugal, por exemplo, também podem interferir nas condições de vida dos idosos. ${ }^{(12)}$

A intervenção cirúrgica que remove a morbidade relativa à catarata possui impacto relevante na percepção do idoso da avaliação daquilo que a pessoa idosa já conseguiu realizar e suas perspectivas para o porvir. Tais fatos são comprovados, também, no domínio da Participação Social, ante ao aumento do escore nos idosos que tinham mais de 69 anos, nas mulheres, nos que tinham religião, nos casados, naqueles que moravam com a família, nos analfabetos e nos que afirmaram saber ler e escrever.

As informações aqui expostas são relevantes para entender que, após a cirurgia de catarata, o aumento do escore na Participação Social dos idosos que tinham mais de 69 anos, ou seja, com idade já bem avançada e propensos a diversas variáveis, pode prejudicar sua saúde, com destaque para as mulheres. A ausência do fator limitante da catarata possui alto impacto no desempenho das atividades exercidas em comunidade nas pessoas idosas do sexo feminino, notadamente nas que têm religião e moravam com a família.

Em pesquisa sobre qualidade de vida de idosos frequentadores de uma universidade aberta da maior idade e a aptidão funcional deles, sugere-se aumento de matérias relacionadas à promoção da saúde e à atividade física, para conscientizar sobre a relevância da manutenção e da melhora da autonomia funcional das pessoas de terceira idade..$^{(22)}$

As sugestões de aumento da realização de atividades físicas e de adoção de práticas de promoção à saúde, passíveis de aplicação no cotidiano dos pesquisados, poderiam também colaborar para o aumento da qualidade de vida e a maior autonomia sensorial e funcional após a cirurgia de catarata.

As limitações da presente pesquisa estão relacionadas à amostragem, ponto superável, por exemplo, com posteriores estudos em localidade com maior número de habitantes, colaborando para a difusão do conhecimento relativo ao tema em diversas localidades do país. 


\section{CONCLUSÃO}

Foi possível traçar um perfil epidemiológico dos pacientes idosos submetidos à cirurgia de catarata. Embora não seja possível afirmar que houve aumento na qualidade de vida do idoso após a submissão à cirurgia por meio do escore geral, o fato revela-se coerente com os demais resultados, à medida em que se denotam as características pessoais e clínicas dos idosos avaliados.

Quanto aos aspectos clínicos, identificou-se que os idosos avaliados possuíam outros problemas de saúde e apresentavam os sintomas da catarata há mais de 1 ano, atrapalhando os afazeres diários. Ainda que tenha sido resolvida a questão relativa à catarata portada pelo idoso, por meio da intervenção cirúrgica, outras variáveis inerentes à velhice podem contribuir para a redução da percepção da qualidade de vida.

Revelou-se, ainda, um perfil sociodemográfico da população entrevistada, que, no caso, possuía baixa capacidade econômica, relações sociais restritas, limitado acesso a informação e, por consequência, ao conhecimento, bem como às atividades físicas, características capazes de influenciar negativamente na saúde dos indivíduos da amostra.

Também verificou-se que, após a cirurgia, houve aumento no escore das Atividades Passadas, Presentes e Futuras e no escore da Participação Social e diminuição do domínio Funcionamento do Sensório.

A realização da cirurgia de catarata proporcionou maior satisfação dos idosos que a ela foram submetidos com as conquistas obtidas e, além disso, esperança na concretização dos objetivos futuros. Os indivíduos avaliados se mostraram mais satisfeitos para participarem das atividades da comunidade à qual pertenciam.

Os resultados da presente pesquisa podem ser utilizados na elaboração de políticas públicas de conscientização, por intermédio de atos e campanhas do Ministério da Saúde e das Secretarias de Saúde estaduais e municipais, contribuindo para a elevação da qualidade de vida das pessoas idosas com deficiências visuais, notadamente a catarata, visando ao tratamento e, se possível, à extirpação desse fator limitante em maior número de idosos.

\section{REFERÊNCIAS}

1. Menezes C, Vilaça KH, Menezes RL. Quedas e qualidade de vida de idosos com catarata. Rev Bras Oftalmol. 2016;75(1):40-4.
2. Instituto Brasileiro de Geografia e Estatística (IBGE). Estatísticas sociais. projeção da população 2018: número de habitantes do país deve parar de crescer em 2047. [citado 2019 Fev 17]. Disponível em: <https:// agenciadenoticias.ibge.gov.br/agencia-sala-de-imprensa/2013-agenciade-noticias/releases/21837-projecao-da-populacao-2018-numero-dehabitantes-do-pais-deve-parar-de-crescer-em-2047>.

3. Macedo BG, Pereira LS, Rocha FL, Castro AN. Medo de cair e qualidade de vida em idosos com catarata. Rev Bras Geriatr Gerontol. 2013;16(3):569-77

4. Pereira MC, Krieger MA, Mariushi AC, Moreira H. Perfil epidemiológico de pacientes com catarata traumática no Hospital de Olhos do Paraná. Rev Bras Oftalmol. 2012;71(4):236-40.

5. Menezes RL, Bachion MM. Condições visuais autorrelatadas e quedas em idosos institucionalizados. Rev Bras Oftalmol. 2012;71(1):23-7.

6. Seidl EM, Zannon CM. Qualidade de vida e saúde: aspectos conceituais e metodológicos. Cad Saude Publica. 2004;20(2):580-8.

7. Yin RK. Qualitative research from start to finish. 2nd ed. New York: The Guilford Press; 2016.

8. Power M, Quinn K, Schmidt S; WHOQOL-OLD Group. Development of the WHOQOL-old module. Qual Life Res. 2005;14(10):2197-214.

9. Fleck MP, Chachamovich E, Trentini CM. Projeto WHOQOL-OLD: método e resultados de grupos focais no Brasil. Rev Saude Publica. 2003;37(6):793-9.

10. Paradella R. Número de idosos cresce $18 \%$ em 5 anos e ultrapassa 30 milhões em 2017. Agência IBGE Notícias. [citado 2019 Fev 17]. Disponível em: <https://agenciadenoticias.ibge.gov.br/agencia-noticias/2012-agenciade-noticias/noticias/20980-numero-d e-idosos-cresce-18-em-5-anos-eultrapassa-30-milhoes-em-2017>.

11. Jesus IT, Diniz MA, Lanzotti RB, Orlandi FS, Pavarin SC, Zazzetta MS Fragilidade e qualidade de vida de idosos em contexto de vulnerabilidade social. Texto Contexto Enferm. 2018;27(4):e4300016.

12. Ferreira LK, Meireles JF, Ferreira ME. Avaliação do estilo e qualidade de vida em idosos: uma revisão de literatura. Rev Bras Geriatr Gerontol. 2018;21(5):616-27.

13. Chatziralli I, Mitropoulos P, Parikakis E, Niakas D, Labiris G. Risk factors for poor quality of life among patients with age-related macular degeneration. Semin Ophthalmol. 2017;32(6):772-80.

14. Marques LP, Schneider IJ, d'Orsi E. Quality of life and its association with work, the Internet, participation in groups and physical activity among the elderly from the EpiFloripa survey, Florianópolis, Santa Catarina State, Brazil. Cad Saude Publica. 2016;32(12):e00143615.

15. Miranda LC, Soares SM, Silva PA. Qualidade de vida e fatores associados em idosos de um Centro de Referência à Pessoa Idosa. Ciênc Saúde Coletiva. 2016;21(11):3533-44.

16. Santana TS, Ávila MP, Isaac DL, Tobias GC, Paranaguá TT. Impacto da facectomias na qualidade de vida de idosos atendidos em campanha assistencial de catarata. Rev Eletr Enferm. 2017;19:1-10.

17. Adamo CE, Esper MT, Bastos GC, Sousa IF, Almeida RJ. University of the third age: the impact of continuing education on the quality of life of the elderly. Rev Bras Geriatr Gerontol. 2017;20(4):545-55.

18. Pinheiro SB. Avaliação do equilíbrio e do medo de quedas em idosos antes e após a cirurgia de catarata senil [dissertação]. Brasília (DF): Universidade Católica de Brasília; 2014.

19. Paula TA, Amigo MH, Avozani ML, Mine A. Facoemulsificação com uso de gancho de íris em paciente com iridosquise. Rev Bras Oftalmol. 2011;70(3):188-90

20. Paz LP, Borges LL, Marães VR, Gomes MM, Bachion MM, Menezes RL. Fatores associados a quedas em idosos com catarata. Ciênc Saúde Coletiva. 2018;23(8):2503-14.

21. Chang VC, Do MT. Risk factors for falls among seniors: implications of gender. Am J Epidemiol. 2015;181(7):521-31.

22. Rocha RE, Mineiro L, Boscatto EC, Mello MF. Aptidão funcional e qualidade de vida de idosos frequentadores de uma universidade aberta da maior idade. J Phys Educ (Maringá). 2016;27(1):e2725. 